
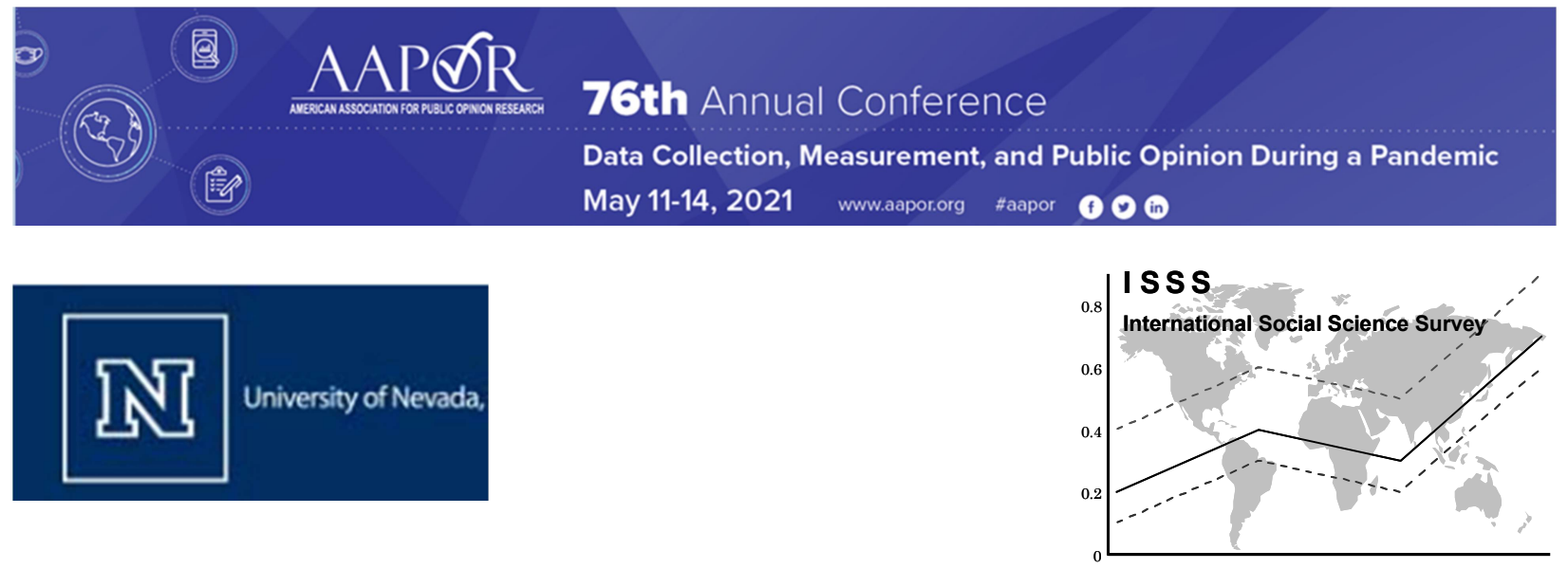

\title{
LET'S SPEND THE NIGHT TOGETHER: A Challenge for Medically Optimal Coronavirus Social Distancing Policies
}

\author{
MDR Evans, \\ Department of Sociology and Nevada Agricultural Experiment Station, \\ University of Nevada, Reno \\ Jonathan Kelley, \\ International Social Science Survey \& University of Nevada, Reno \\ Sarah Kelley, \\ Department of Data Science and Innovation, Child Trends
}

December 2020

Let's spend the night together

Now I need you more than ever

Mick Jagger \& Keith Richards, Rolling Stones, 1967

\begin{abstract}
Public health efforts to slow the COVID-19 epidemic and protect the vulnerable have focused on reducing interpersonal contact and increasing interpersonal physical distance. They have achieved considerable success, with over three-quarters of Americans reporting that they follow all the recommended precautionary practices of social distancing, so it is likely that, at least, they follow most of them most of the time. But there is an elephant in the room: Overnighting - staying overnight at someone else's home or having them stay overnight at your place is rarely, if ever, mentioned publicly. But we will show that this is a widespread practice in American society, throughout the epidemic thus far. In the past month, over half of all Americans had at least one overnight guest and about the same number (but not always the same people) spent at least one night in the past month at somebody else's home. The consequences are striking: People who overnight are more than twice as
\end{abstract}


likely to report that they have had COVID-19. Who overnights? The practice is much more common among young adults (over 60\% for those 25-34) than among their seniors (dipping below $30 \%$ for those age 65 and over). Moreover, risk seekers are hugely more likely to overnight than are their otherwise similar risk averse peers. Data: International Social Science Survey, Round 21, Cohorts 1-5, collected April July 2020, N=4,815. Methods: Descriptive statistics and multiple regression.

\author{
Paper to be presented to the \\ Annual Conference of the American Association for Public Opinion Research, \\ Friday, 14 May, 2021 at 11:30am EDT.
}

Key words: COVID-19, coronavirus, social distancing, overnight, sleep over, sexual behavior, visiting, age, multiple regression,

\title{
INTRODUCTION
}

"Social distancing" is the sovereign defence against COVID-19, as it is also against many other respiratory viruses. Throughout the world during the current coronavirus pandemic, medical authorities have recommended - and many governments attempted to compel - "social distancing". "Social distancing" has entered ordinary Englishlanguage speech as an umbrella name for recommended precautionary practices that limit contact with respiratory droplets spewed into the air when an infected person coughs, sneezes, talks or sings. These droplets can be breathed in by other people or can land on surfaces that are touched by other people who then touch their own mucus membranes and are thereby infected.. The airborne droplet issue is especially acute if the spew-receiver is in close contact with the infected person (within about 6 feet) and in enclosed spaces. Most common social distancing measures are wearing masks; staying 6 feet away from others; not shaking hands; washing hands after being out in public; avoiding crowded places (churches, bars, gyms, theatres, sports venues); refraining from visiting friends in their homes or letting them visit in your home; and working at home instead of outside the home. Some authorities restrict the "social distancing" term to maintaining 6 -foot physical distances from people who are not members of your household, but popular speech seems also to include the other standard precautionary measures under this rubric. In extreme situations (common throughout the world in the spring and early summer of 2020) was self-quarantining at home, going out only minimally if at all.

Public health policies for dealing with the coronavirus epidemic usually implicitly assume that establishing consistent social distancing behavior will in time control the epidemic. The image they seem to have in mind is a world of nuclear families (selfsufficient singles, married couples, families with children), each living in a separate home and only interacting with people outside the family in stereotypical ways commuting to work; at work outside the home; and out in public for medical care, 
exercise, shopping, at restaurants, bars, or parties, or socializing with friends (Hossain 2020, Manzo 2020). Publicly recommended distancing policies cover all these.

In practice, people in the US and in many other nations have mostly endorsed these distancing policies even at the beginning of the epidemic (McFadden et al. 2020) and throughout its peak in the US. They have largely, although by no means entirely, practiced them, averaging about three quarters of the maximum distancing (Azlan et al. 2020). More on that presently. This is typically at some cost - sometimes at great cost - in personal unpleasantness and financial loss - and the distress of deprivation of in-person contact seems to fall especially heavily on young unattached adults throughout the world (Hou et al. 2020, Lehmiller et al. 2020, Varshney et al. 2020, Wang et al. 2020).

But these public health injunctions discreetly ignore the elephant in the room: Overnighting. For example, the key CDC public information internet page on social distancing mentions neither having overnight guests, nor spending the night at other people's homes, nor dating, nor sexual behavior (CDC 2020). Public health authorities would doubtless hold that refraining from spending the night together is implicitly covered by the other injunctions (e.g. refraining from home visits with friends). But that mild injunction, only one among many, is insufficient to the task. People view night almost as another world where different rules apply (Melbin 1978, Zerubavel 1985). Forgoing daytime visiting and dinner in-person is a hardship, but, even early in the epidemic, had been partially compensated by a strong upsurge in the use of internet apps that facilitate video visits among family and friends (Eagle 2020). By contrast, spending the night together, especially spending it in bed together, is different: a delight when all is well - and a delight very hard to forgo voluntarily even though innovative people have tried many online approximations (Lehmiller et al. 2020). At least through May 2020, it seems that young, unattached people's in-person sexual frequency had only declined slightly (Lehmiller et al. 2020). Unfortunately, overnighting - whether romantic, or couch surfing, or sleeping over at a family member's home or other -- also entails a high risk of infection if the pairing is unlucky with respect to the new coronavirus, as this article will show.

We find that, even at the midst of the coronavirus pandemic, roughly half of all Americans had, within the month, someone spend the night at their home, or themselves spent the night at someone else's house, or did both. And this usually multiple times in the month.

Social distancing this is not. A major challenge to public health policy it is: The spread of coronavirus through overnighting is a major risk. In fact, it is among the largest potentially controllable risks in the US under present circumstances.

\section{DATA}

Data are from five waves of our International Social Science Survey's (ISSS's) ongoing Round 21 in the United States with nine surveys between mid-April and late September, 2020 and more subsequently. 
In relation to the new coronavirus, reported infections per day in the US were near zero in mid-March, rising sharply to about 20,000 per day at the beginning of April and continuing their strong rise to a peak of a little over 30,000 by mid-April (all infections data are from the Johns Hopkins University database; numbers in text are from a 7 day running average to smooth out the sharp day-of-week fluctuations presumably due to reporting constraints). Our first survey was a few days after the peak, with a little over 30,000 new infections daily. The second survey a couple of weeks later was when new infections were fractionally lower, just on 30,000 cases a day. The third survey was after the decline was clearly underway at the end of May, with a little over 20,000 new cases per day. Subsequent surveys continued through the summer ;and up to late September; we have not yet covered the October rise in infections (our 10th survey, is not yet processed as this is written).

The key "others stay overnight at your house" question was asked in all nine surveys. The parallel question on "you staying overnight at someone else's home", and two follow-up questions on the loss of utility from not being able to stay overnight were included beginning only in the May survey, after we discovered how widespread sleepovers are throughout American society and how seriously it increases the risk of COVID-19.

All of the surveys so far are Amazon 'Mechanical Turk' internet-based samples. These can be conducted without risk of contracting coronavirus and, moreover, are swift, convenient, and cost effective. Internet samples of this sort have been widely used during the pandemic, for example (Imhoff and Lamberty 2020, Lehmiller et al. 2020, Wang et al. 2020, Yan et al. 2020)

Mechanical Turk samples have proven remarkably accurate for multivariate analyses (Clifford, Jewell and Waggoner 2015, Cornesse and Blom 2020, Springer et al. 2016, Weinberg, Freese and McElhattan 2014), notably for political affiliations and attitudes (Clifford, Jewell and Waggoner 2015) and for science-related issues (Evans and Kelley 2011). We, too, have found that when comparing our ISSS 2017-2019 internet samples to face-to-face samples in the US and dozens of other countries (from the International Social Survey Programme's highly regarded "Inequality" series).

A disadvantage is that estimation of population means in internet samples may need to be weighted because they typically over-represent younger and more highly educated people. In multivariate analysis, of course, inclusion in the model of the variables on which the sample is not representative corrects for the compositional bias (Berk 1983), so age and education are included in all out models.

Our internet samples appear to be representative of the general population on issues related to the new coronavirus. For example, the percent proposing that coronavirus entered the human population when they "caught it from wild animals in a market in Wuhan, China", the $45 \%$ in our ISSS surveys is virtually identical to the $43 \%$ reported by Pew for their April survey on the subject (Schaeffer 2020). It is reassuring that our results - based on a non-probability Mechanical Turk sample - so closely mirror those of Pew's carefully selected nationally representative panel. 


\section{MEASUREMENT}

Details on the key "spend the night together" questions are elaborated just below in the "Description" section.

All the multiple-item scales described below have good measurement properties, closely approximating the classical measurement model (Bollen 1989, Treiman 2009) with high correlations among items in each scale, scale items having very similar correlations with relevant criterion variables, and appropriately high loadings in factor analyses (see Appendix A or the supplementary materials).

For scale items and in regression analyses, answer categories are scored in equal intervals ('Likert' scoring) between 0 and 1 (sometimes between 0 and 100). This gives more intuitively clear means and metric regression coefficients than scorings of 1-2-34-5. Although superficially ordinal, the classic Likert answer categories were designed to be treated as quantitative variables with categories spaced at equal intervals (Likert 1932) and, in practice, respondents treat them like that (Carifio and Perla 2007). For standardized coefficients ( $\mathrm{R}^{2}$, betas, etc.) this scoring is mathematically identical to the 1-2-3-4-5 scoring or any other equal interval scoring (Nowak 2019). Metric results (means, standard deviations, regression coefficients) differ only by a simple scale factor.

Our multiple-item scales are constructed by simply averaging the component items, because such scales replicate better than those based on the factor loadings which tend to overfit (Bollen 1989). If some items in a scale were not answered (rare), the average of the remaining items was used.

Distancing is a five-item scale of how often respondent practices recommended distancing behavior: staying 6 feet away from others when out in public, not shaking hands, washing hands often, avoiding parties, avoiding going to other people's house or having them come to your house. Answers were in 7 categories from "always" (scored 1) through "never" (sored 0). Alpha reliability is a very satisfactory .84. The battery begins:

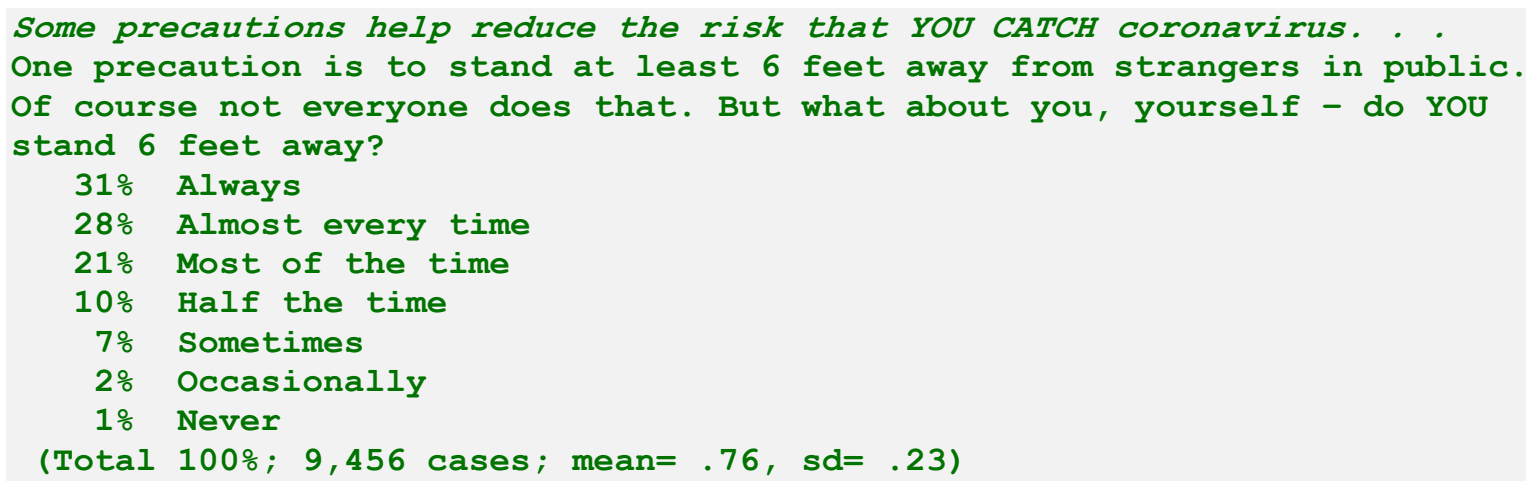

Standing 6 feet away is a typical distancing behavior and its mean of .76 (on a zero to 1 scale) is also typical for all five items. In this sense, Americans during the COVID-19 pandemic are distancing around three-quarters of the time. 
Wearing masks is a three-item scale covering willingness to wear a face mask while shopping, at work, and other times when out in public; Alpha=.85. Statistical analysis suggests wearing masks is highly correlated with, but nonetheless somewhat distinct from, other distancing behaviors (identifying reference suppressed for review).

Having been infected with coronavirus is measured (but that only roughly and approximately) largely with a single question. Infection is a difficult thing to ascertain in a sample survey because of Covid-19's varied, often trivial, and sometimes entirely absent symptoms, compounded by the stress of living through the epidemic. Our measure is no more than a rough beginning. The question:

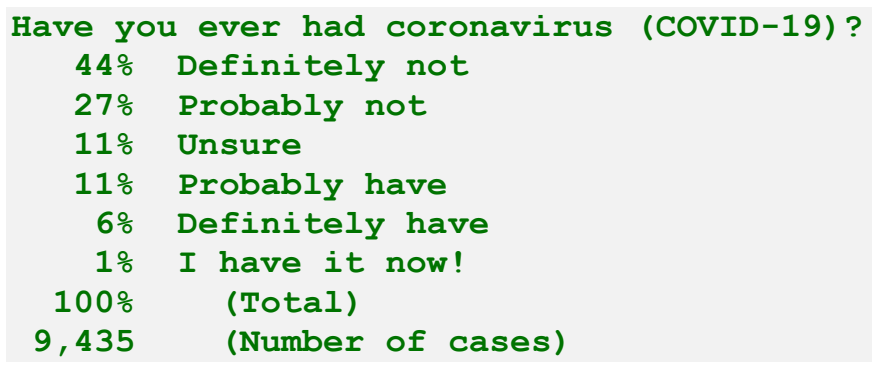

These numbers seem high, but the $6 \%$ who "definitely" have had it or have it "now" that we find is only moderately higher than the 5\% for March 25 (Imhoff and Lamberty 2020), a month before our surveys began. We refined these answers on the basis of an explicit question about having been tested positive for IgG antibodies (showing a prior infection) or alternatively testing positive for the presence of the virus (showing a then-current infection), treating both as showing respondent has definitely had coronavirus. With these adjustments and assuming equal intervals between answer categories, our rough approximation is $18 \%$ definitely having had coronavirus (scored 1), 7\% likely to have had it (scored 0.75 ), $9 \%$ with a $50-50$ chance of having had it (scored 0.50 ), $24 \%$ possibly having had it (scored 0.25 ), and $42 \%$ definitely not having had it (scored 0). Treating those as a (very approximate, likely exaggerated) probability of having been infected, the mean is 0.33 and the standard deviation 0.38. This approximation is used in the analyses that follow.

Other general population surveys have obtained similar, probably similarly exaggerated, figures - for example an AP-NORC poll in May reported 20\% for "you or a close friend or relative" having been so diagnosed "by a health care provider", similar to the $17 \%$ (raw) or $21 \%$ (adjusted) we have for "definitely" or "probably" believing they have had coronavirus. UK results are similar (Thornlow 2020). Recent estimates from the CDC suggest close to $10 \%$ have had it.

Demographic and background variables are measured conventionally. Male, Black and Hispanic are dummy (indicator) variables; the reference (omitted) group is White and Other. Education is in years of schooling and university. Occupational status is the new SIOM scores (Kelley and Evans, 2018), known to be largely equivalent to, and in mobility analyses often better than, the excellent TreimanGanzeboom ISEI scores. Family income is in thousands of US dollars. Democratic is vote intention in the 2020 US presidential election (strong Democratic $=1$; leaning Democratic $=.75$; Independent $=.50$; leaning Republican $=.25$; strong Republican $=0$ ), 
included as research even from the very beginning of the epidemic suggests considerable political polarization of COVID-19-related attitudes and perceptions (Matthewman and Huppatz 2020, Pennycook et al. 2020, April 14).

\section{DESCRIPTION}

The key "night together" question, asked in all nine surveys, is:

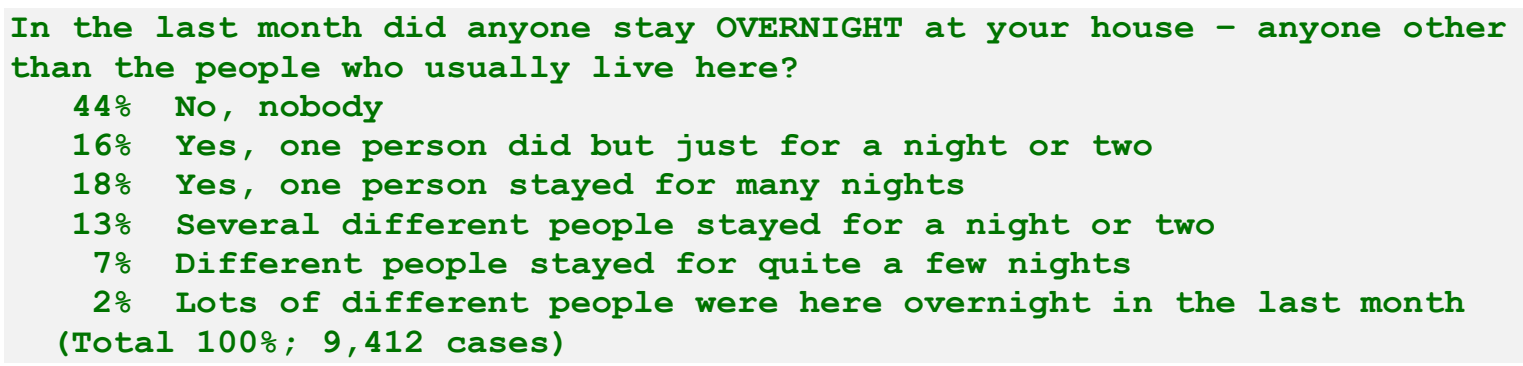

After discovering the (astonishingly) large number of people who had overnight guests - and that in the midst of an epidemic - after the first two surveys we also asked the reciprocal question:

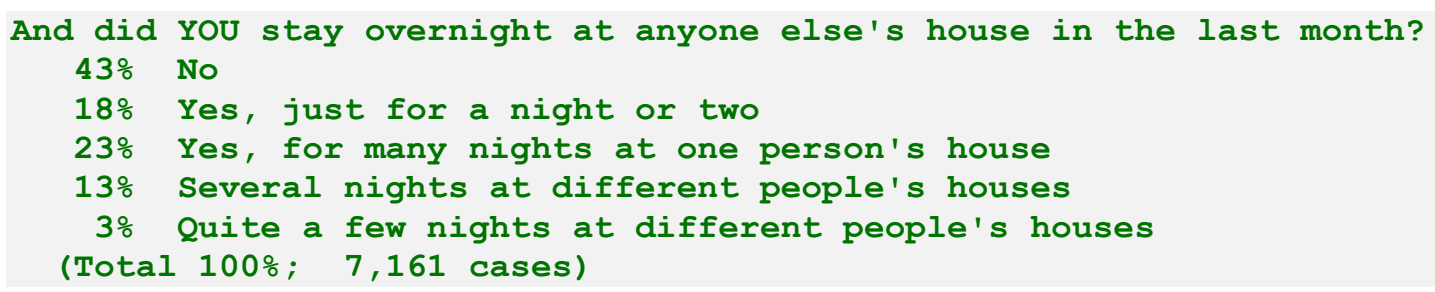

Thus, a majority, $57 \%$, spent at least one night at someone else's place in the last month. Fully 16\% report multiple overnighting with more than one person. Thus, the stereotypic image of a world of separate, largely isolated units (singles, married couples, families with children), each living in a separate residence, only interacting with outsiders in urban civic and associative ways - commuting to work; at work; and out in public for medical reasons, exercise, shopping, at restaurants, bars, or parties; or socializing with friends - is a daytime and early evening image. Social distancing mandates are designed for that familiar, overt world, isolating one unit from another, so hindering the spread of coronavirus (and other infectious diseases).

But things are different at night. Night is a time of intimate contacts between people normally living in separate residences. Sleepovers are, of course, not all romantic: They include visits by family and friends and overnight visitors to housemates or other family members. Night can be a wonderful time for people, but sometimes a risky one. For coronaviruses it is a happy time.

\section{OVERNIGHTING'S INFECTIOUS CONSEQUENCES}

As far as we can tell from our (admittedly rough) question on having been infected, having overnight guests roughly doubles the risk of getting coronavirus from a 
(probably exaggerated) $18 \%$ to around $40 \%$ or $50 \%$ (Figure \&\&\&1). This is a maximum figure, without adjusting for correlated factors, a matter to which we will return shortly.

[Figure \&\&\&1 about here]

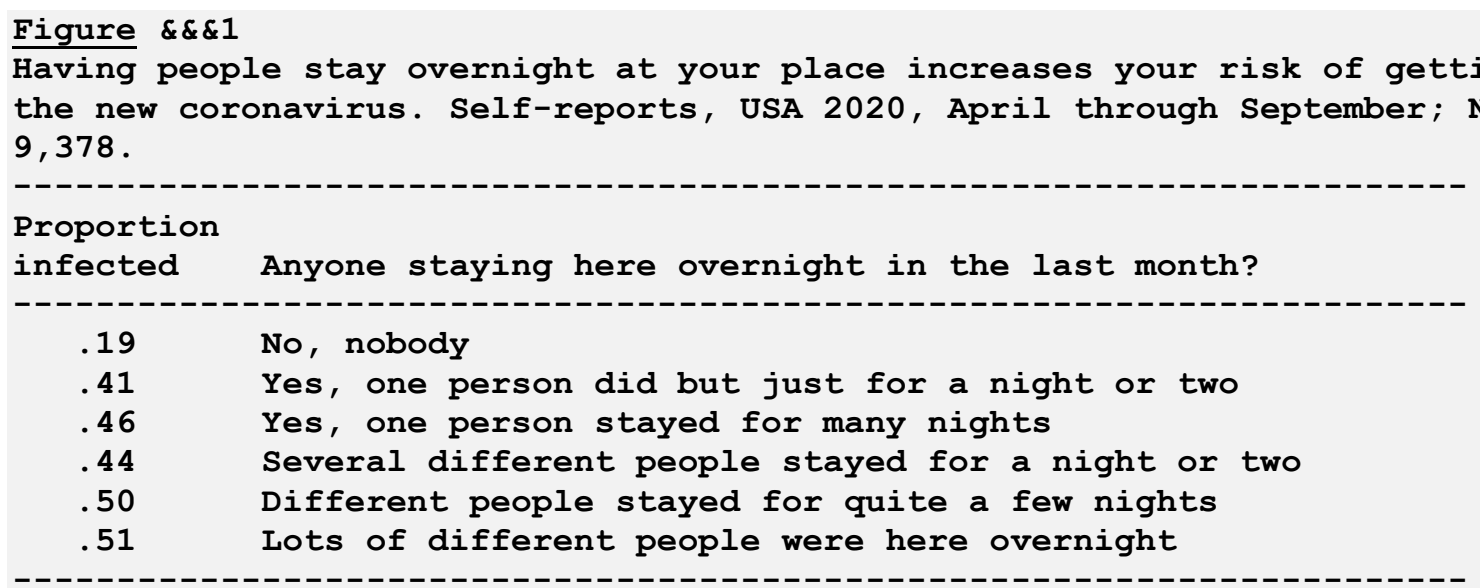

Across all the various patterns of overnighting (one person for many nights; different people for a few nights, etc.) the greatest increase in risk is between having no overnight guests and having any. Hence, it is reasonable to dichotomize the item between "no" (scored 0) and "some overnighting" (scored 1) - this despite the general and well-known analytic weakness of dichotomous items (Bollen and Barb 1981, Nowak 2019).

That is the effect of someone staying overnight at your place. The effect of your sleeping over at someone else's place is about the same (Figure \&\&\&2). It more than doubles the risk from $17 \%$ to something around $40 \%$ or $45 \%$. It is therefore sensible to dichotomize that item between "no" (scored 0) and "some overnighting" (scored 1).

[Figure \&\&\&2 about here]

\begin{tabular}{|c|c|}
\hline $\begin{array}{l}\text { Your stayin } \\
\text { getting the }\end{array}$ & $\begin{array}{l}\text { g overnight at someone else's place increases your risk of } \\
\text { new coronavirus. USA 2020, May through September; } N=7.130\end{array}$ \\
\hline $\begin{array}{l}\text { Proportion } \\
\text { infected }\end{array}$ & You stay overnight at someone else's house? \\
\hline $\begin{array}{l}.19 \\
.41 \\
.46 \\
.48 \\
.48\end{array}$ & $\begin{array}{l}\text { No } \\
\text { Yes, just for a night or two } \\
\text { Yes, for many nights at one person's house } \\
\text { Several nights at different people's houses } \\
\text { Quite a few nights at different people's houses }\end{array}$ \\
\hline
\end{tabular}

For reliability and parsimony in the analysis that follows we average the two "overnighting" items in those surveys where both were asked. (In the two early surveys where only the one question was asked, we use it alone.) This gives a reliable scale (Alpha $=.89)$ : 


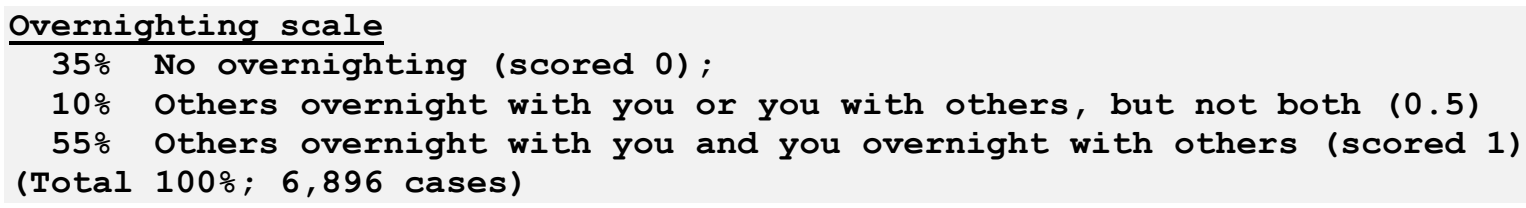

\section{OVERNIGHTING, DAYTIME DISTANCING, AND CORONAVIRUS}

People who let others stay overnight, or who themselves stay overnight at somebody else's place, or do both, are much less careful about social distancing during the day (Figure \&\&\&3, column 2, in green). They are less likely to stay 6 feet away from strangers in public $(\mathrm{r}=-.33)$; less likely to avoid shaking hands $(\mathrm{r}=-.48)$ or to avoid parties $(\mathrm{r}=-.36)$ or to avoid visiting during the day $(\mathrm{r}=-.35)$.

So, their behavior is more risky during the day as well as during the night.

[Figure \&\&\&3 about here]

\begin{tabular}{|c|c|c|c|c|c|c|c|}
\hline & $\begin{array}{l}\text { Enfected } \\
\text { (1) }\end{array}$ & $\begin{array}{l}\text { Overnight } \\
\text { (2) }\end{array}$ & $\begin{array}{c}6 \text { feet } \\
\text { (3) }\end{array}$ & $\begin{array}{c}\text { Shake } \\
(4)\end{array}$ & $\begin{array}{l}\text { Wash } \\
\text { (5) }\end{array}$ & $\begin{array}{l}\text { Visit } \\
(6)\end{array}$ & $\begin{array}{r}\text { Mask } \\
(7)\end{array}$ \\
\hline Overnighting & 0.3560 & 1.0000 & & & & & \\
\hline Conventional dis & Eancing: & & & & & & \\
\hline Stay 6 feet away & -0.2122 & -0.2954 & 1.0000 & & & & \\
\hline Not shake hands & -0.2751 & -0.4643 & 0.5369 & 1.0000 & & & \\
\hline Wash hands & -0.3125 & -0.4439 & 0.5191 & 0.5769 & 1.0000 & & \\
\hline Not visit & -0.1801 & -0.2995 & 0.4224 & 0.4580 & 0.4469 & 1.0000 & \\
\hline Wear mask & -0.1957 & -0.2659 & 0.4495 & 0.4422 & 0.4927 & 0.3683 & 1.0000 \\
\hline Distancing scale & -0.3104 & -0.4755 & 0.7542 & 0.8038 & 0.7923 & 0.7588 & 0.5713 \\
\hline
\end{tabular}

That risky behavior during the day does have the forseeable consequences, on average and net of other things, the less you daytime distance, the more likely you are to get COVID-19 ( $\mathrm{r}=.37$; Figure \&\&\&4, top panel, in red). Just which daytime distancing measures are the bst protection, and for whom, is a fraught question, since distancing in one way (for example, staying at least 6 feet away) is strongly correlated with taking precautions in other ways (for example not shaking hands (Figure \&\&\&3, figures highlighted in blue). In this example the correlation is $r=.58$ (column 3 , second line of the "Daytime distancing" panel). So, it is statistically difficult to say which of these highly correlated distancing precautions makes for safety and by how much.

Fortunately for our issues we do not need to know the details, just the overall outcome. For that we can combine the distancing precautions, simply averaging them into a single (highly reliable) distancing scale (Figure \&\&\&3, last row).

For our analysis, this summary scale captures all that we can capture about daytime distancing and coronavirus infection (Figure \&\&\&4, first panel, in red). Taking the five items separately does not add any statistically significant information. 
[(Figure \&\&\&4 about here]

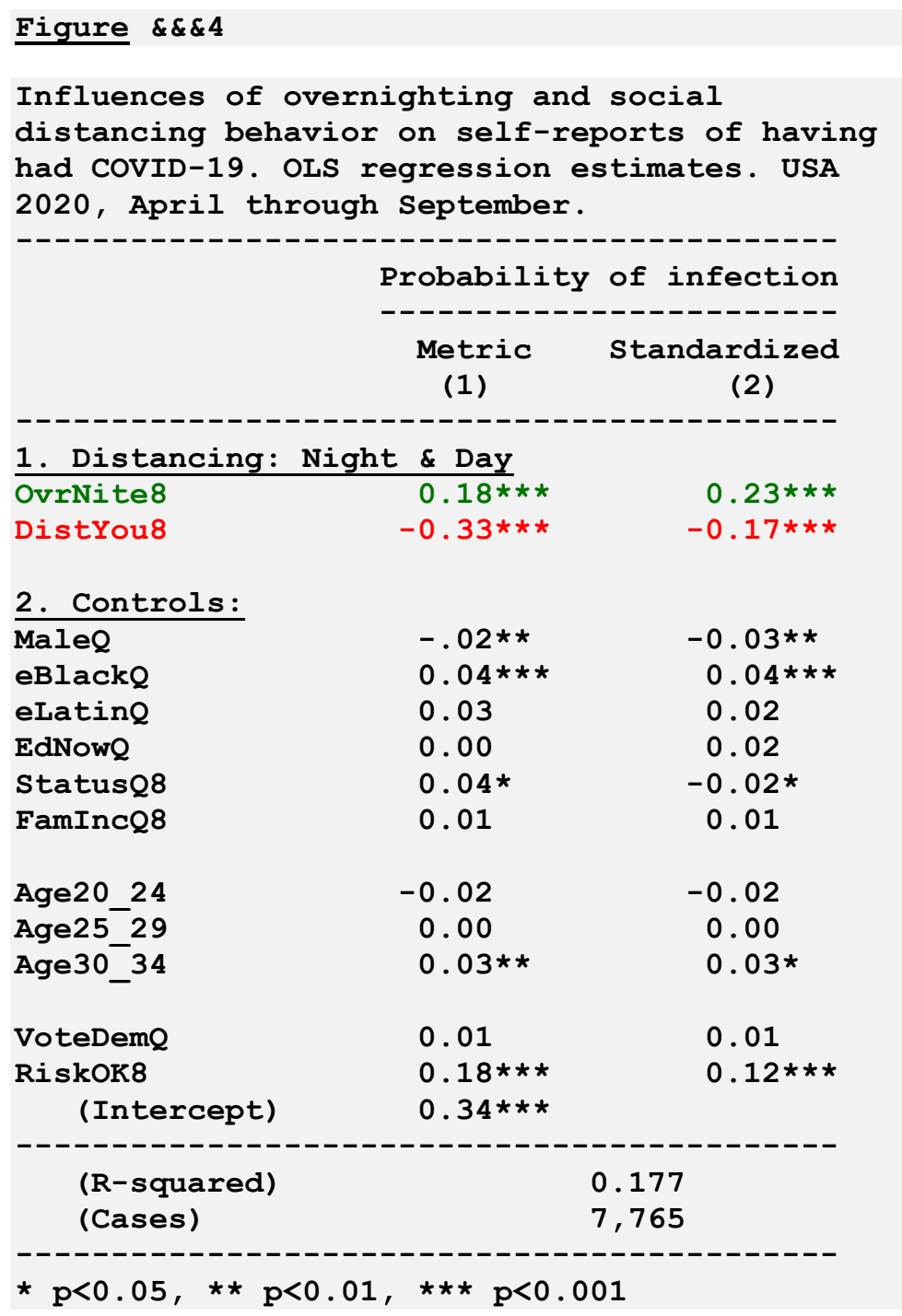

Subject to the many uncertainties in our self-reported measure of having been infected, this strongly suggests that daytime distancing behavior vastly reduces the chance of catching the new coronavirus. Someone who never distances at all - no 6 feet away, no washing hands, always shaking hands, never avoiding parties or visits - but is otherwise average has a probability of .59 of getting infected. But if they instead distanced faithfully and consistently - always staying 6 feet apart, always washing hands regularly, never shaking hands, never going to parties, never visiting - their probability of getting coronavirus would be only .26. So such extreme distancing reduces the probability of infection by fully .33 points (viz .26 -.59=-.33). The $95 \%$ confidence intervals for these estimates are .23 to .29 for never social distancing and .55 to .63 for always social distancing.

This is a vast difference, amply justifying the familiar and unequivocal medical and public policy recommendations in favor of distancing. 


\section{DISTANCING AT NIGHT}

The same logic shows many of the benefits of daytime distancing can be undone at night (Figure \&\&\&4, first row and first column in Panel 1, in green). This is again subject to the many uncertainties in our self-reported measure of having been infected.

Everything else equal (including daytime distancing) someone who never has overnight visitors, and never visits overnight themselves, has a probability of just .24 of getting infected ( $95 \%$ confidence band .21 to .27). But if they have overnight visitors, and themselves visit overnight from time to time, the probability of infection rises to .42 (confidence band .39 to .45 ), a difference of .18 (viz .42 - .24=.18). That is a large difference, not far off half the difference due to the full suite of daytime social distancing behaviors.

Thus, night is a risky time.

As far as practical policy initiatives go at this stage of the coronavirus pandemic (through August), the night matters at least as much as the day (Figure \&\&\&4, standardized results in column 2, first two rows, in green for overnighting and red for daytime distancing). These standardized regression coefficients compare the estimated impact of a one standard deviation change in overnighting to a one standard deviation change in daytime social distancing.

By that measure overnighting is at least as important as daytime distancing, .23 versus - .17 (that one is positive and the other negative is irrelevant here, just reflecting choice of labels).

The substance behind this is that daytime distancing is already very widely practiced, averaging around .75 on a scale of 0 to 1 . So, there is not all that much room for further improvement. But nighttime distancing is, by contrast, currently around the midpoint on the 0 to 1 scale, so there is still a lot of room for change, for better (from a public health point of view) or for worse.

\section{WHO OVERNIGHTS?}

On the question of who it is that overnights, the short answer is "young adults" (Figure \&\&\&5). Overnighting starts abruptly in the early 20 s, reaches its peak near 30 , and then declines steadily (but not abruptly) through the 40s and 50s.

The big peak of visits in the 20s and 30s are likely to be romantic, for the most part. Of course not all overnight visits are romantic. Some will be relatives; some will be friends; some friends of one's children, dates of one's housemates, and so on.

[Figure \&\&\&5 about here] 


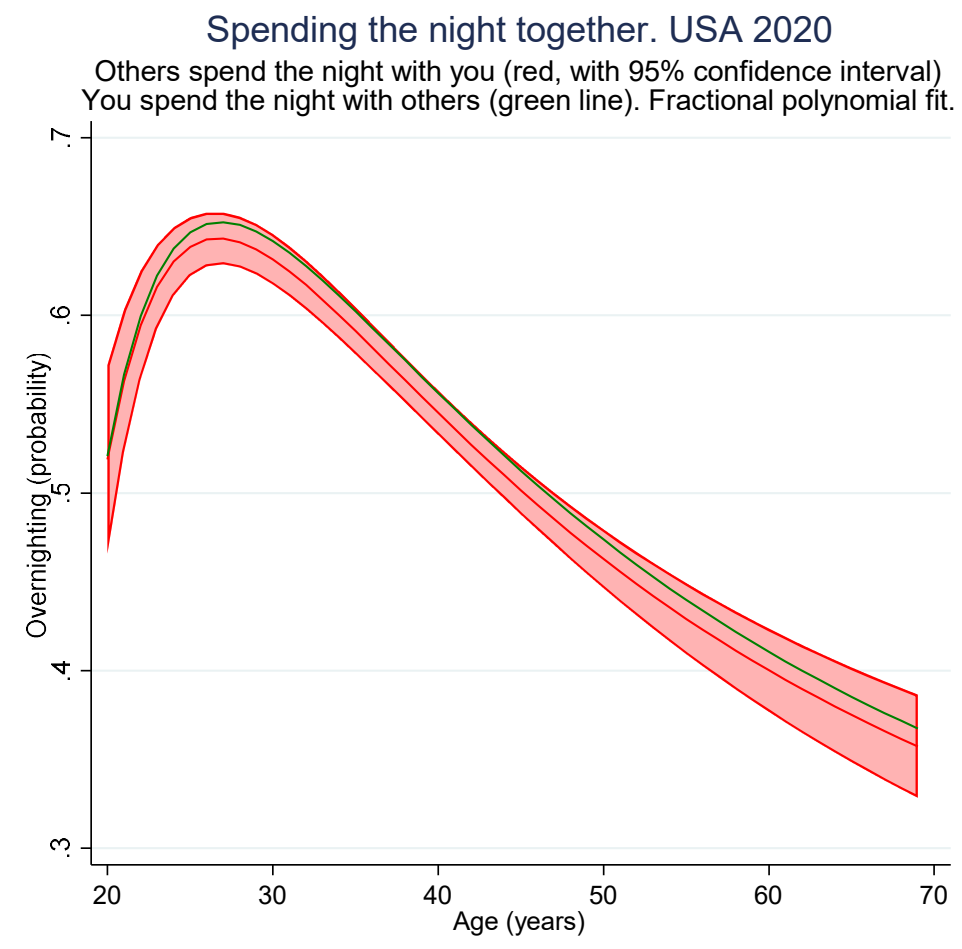

In addition to the striking age profile, there is another way in which overnighters are distinctive: They are very willing to take risks (Figure \&\&\&6, figures highlighted in blue). This is a general risk tolerance - taking risks in driving, in financial matters, and at work, as measured in (Dohmen 2006). If someone is very risk averse (0 on the 0 to 10 scale) they typically have only a $12 \%$ chance of having been infected, all else equal. But if they are risk-seekers (10 on the 0 to 10 scale) they have an $51 \%$ chance of having been infected $(t=223, p<.0001)$. That is a huge difference.

[Figure \&\&\&6 about here]

Apart from age and risk tolerance, there are, at most, weak differences in overnighting. All else equal, men are only fractionally more likely than women to have overnighted. Blacks are 9\% more likely to have overnighted, a significant difference from Whites $(p<.001)$. Hispanics are not significantly different. There are no significant differences between the well educated and those with little schooling, but people with high status jobs (higher professionals and high ranking managers) are $6 \%$ more likely to overnight than are those at the bottom of the status hierarchy (farm workers; $\mathrm{p}<.01$ ). Prosperous people are no different than otherwise comparable poor folk. Democrats are $12 \%$ less likely than Republicans to overnight $(\mathrm{p}<.001)$. 


\section{POLICY: ALLOW OVERNIGHTING DURING THE EPIDEMIC?}

There is a further complication in persuading young people to avoid overnighting, even though the public health benefits are clear. The difficulty is that many people - and not just the young - believe that young people ought to be allowed to date. Dating implies physical intimacy and - in the fullness of time and with the natural evolution of a relationship - eventually sex and spending the night together.

After a series of questions about laws enforcing distancing policies for Americans in general, we asked specifically about young people dating. The state of opinion through September 2020, many months into the pandemic is this:

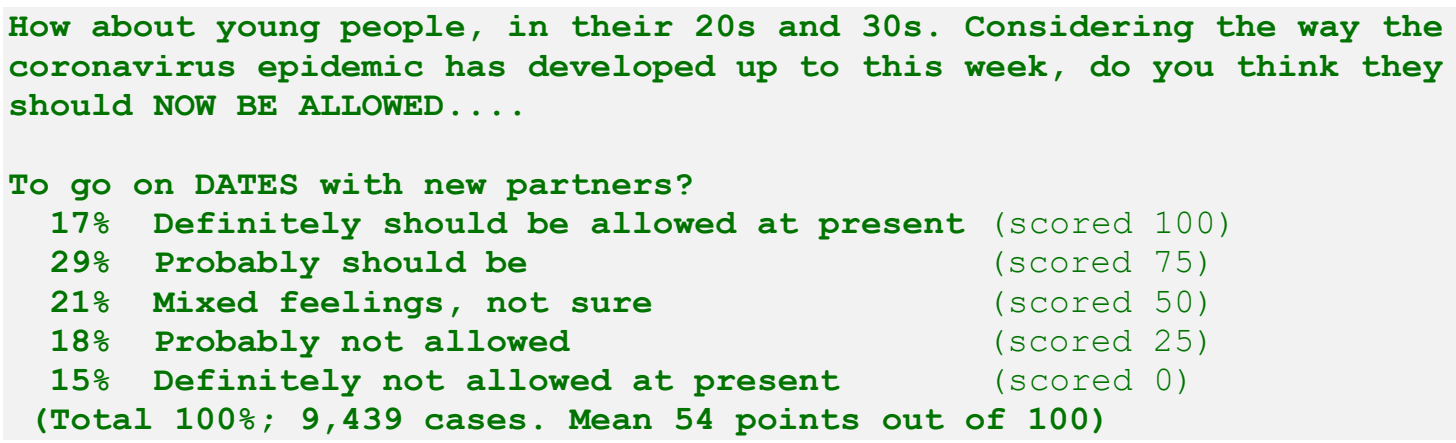

Opinion on dating is almost evenly divided (as of July 2020). Many support the idea close to a majority - while almost as many oppose it, and quite few have mixed feelings. If it had come to a simple yes or no vote, allowing dating would probably have carried the day by a narrow margin (the mean is 54 points out of 100 , fractionally above the 50-50 half way point of "Mixed feelings, not sure").

Thus if we assume that allowing dating in modern America at least implicitly allows for the possibility of eventually spending the night together, this means that there is no strong public support for banning romantic overnight visiting. This is in sharp contrast with opinion on other social distancing measures where the mean averages 68 , not far from "probably should be required by law".

Moreover, support for dating is increasing by nearly one point per week, rising from around 46 in the epidemic's early peak in April to 62 in September $(t=15.9, p<.001)$. This is net of changes in SES and a slow decline in support for daytime distancing (from 79 to 72 points out of 100; regression analysis not shown).

So public health prescriptions to make nights safer from coronavirus face an uphill battle, and one on present trends likely to get harder as time goes on.

\section{DISCUSSION}

\section{Summary}

The results show that overnighting is widespread in American society, even throughout the pandemic thus far, with more than half of adult Americans having at 
least one overnight guest in the past month and a similar number having slept over in another home; almost two-thirds, $63 \%$, did at least one of these things. Regression analysis shows that overnighting is widely diffused socially, with no difference by education, income, or Hispanic ethnicity, and only small differences by political party preference, occupational status, and race. There is a very large and strong difference according to the personality trait of risk aversion: Risk seekers are enormously more likely to overnight than are their risk-averse peers. Age also matters a lot: Adults in their 20 s and early 30 s overnight much more than do otherwise similar people who are older.

The results also show that this is very consequential for catching COVID-19. People who overnight even just once a month more than double their risk of self-reported COVID.

Further, mobilizing social support for policies to reduce overnighting would be difficult as we also find only minority support for policies to discourage young people from going on dates with new partners. Moreover, this support is slowly dwindling over time, so reversing that trend might be an uphill battle.

\section{Implications}

An important consequence of spending the night together is that even complete compliance with the conventional daytime social distancing policies will not be enough to halt the spread of the new coronavirus.

Although overnighting is less common among people who consistently and scrupulously follow daytime social distancing recommendations, it is far from absent (Figure \&\&\&7). Even among people who consistently follow ALL the daytime social distancing restrictions, some $24 \%$ nonetheless have others stay overnight (often several people and many nights) or themselves sleep over at other people's homes (22\%) often for many nights, sometimes with several different people over the course of the month. $25 \%$ do one of these or the other and $20 \%$ do both. These are long-lasting intimate contacts, often in the same bed, are ideal for spreading coronavirus. 


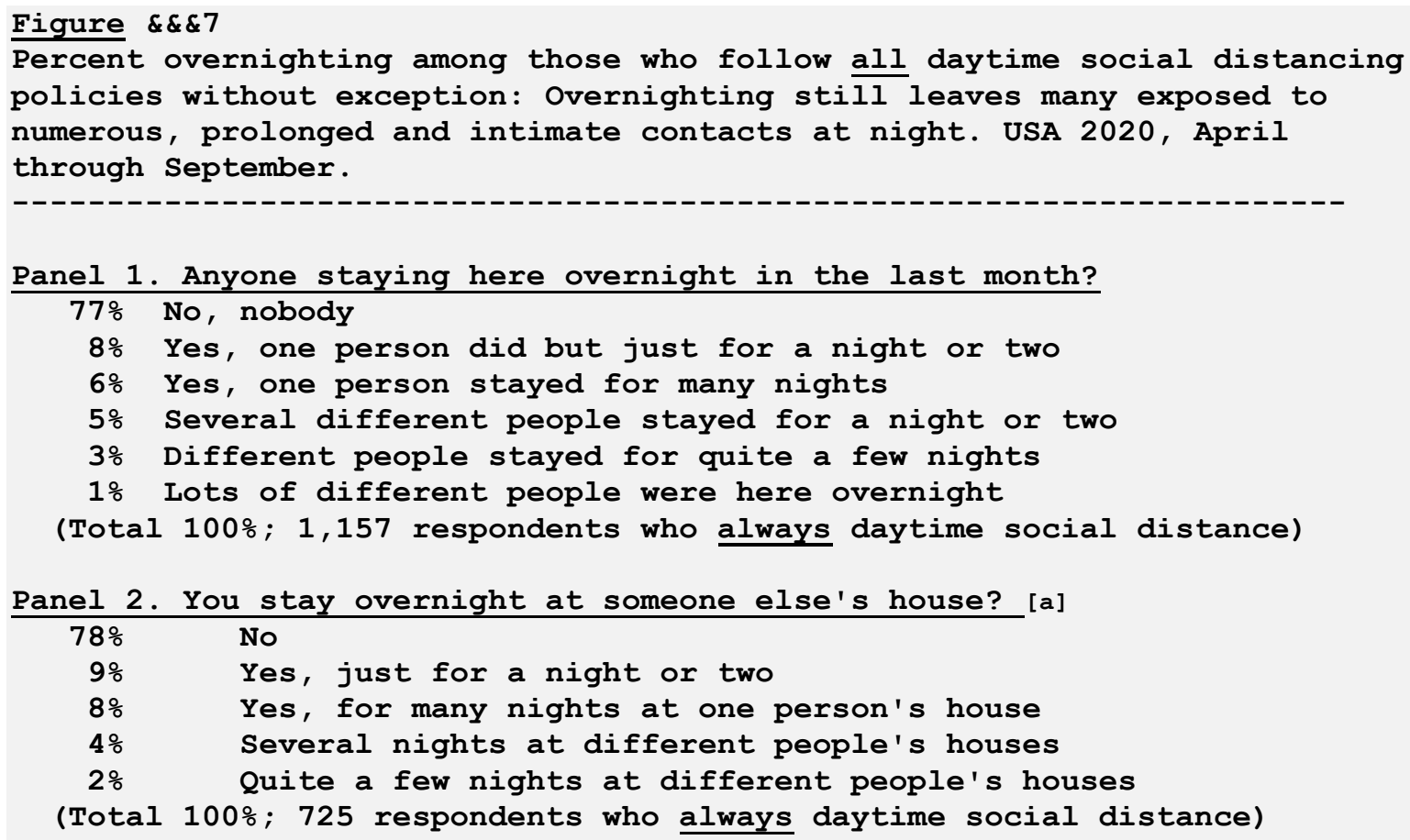

[a] Question not asked In the first two waves of the survey.

The policy implications of these results emphasize reaching out to younger adults and risk-seekers specifically about overnighting, rather than hoping they will follow recommendations against "visiting". Well publicized testimonials from "cool" younger adults who are demonstrably risk-seekers (e.g. well known practitioners of extreme sports) and who caught COVID talking about how they thought they would not get it and how shocked they were when they got it might be among the tactics to deploy here.

An alternative and perhaps more feasible public health policy is to focuse on harm minimization, encouraging (or requiring) overnighters to promptly test themselves for infection and then self-quarrantine if infected.

\section{REFERENCES}

Azlan, Arina A., Mohammad R. Hamzah, Tham J. Sern, Suffian H. Ayub and Emma Mohamad. 2020. "Public Knowledge, Attitudes and Practices Towards Covid-19: A Cross-Sectional Study in Malaysia." PLoS One May 21, 2020:https://doi.org/10.1371/journal.pone.0233668. doi: https://doi.org/10.1371/journal.pone.0233668.

Berk, Richard A. 1983. "An Introduction to Sample Selection Bias in Sociological Data." American Sociological Review 48(3):386-98.

Bollen, Kenneth A. and Kenney H. Barb. 1981. "Pearson's R and Coarsely Categorized Measures." American Sociological Review 46(2):232-39. doi: 10.2307/2094981. 
Bollen, Kenneth A. 1989. "Confirmatory Factor Analysis." Pp. 226-318 in Structural Equations with Latent Variables: Wiley-Interscience.

Carifio, James and Rocco J. Perla. 2007. "Ten Common Misunderstandings, Misconceptions, Persistent Myths and Urban Legends About Likert Scales and Likert Response Formats and Their Antidotes." Journal of Social Sciences 3(3):106-16.

CDC. 2020, "Social Distancing,. July 15, 2020", Atlanta: US Government. Retrieved August 28, 2020 (https://www.cdc.gov/coronavirus/2019-ncov/prevent-gettingsick/social-distancing.html).

Clifford, Scott, Ryan M Jewell and Philip D Waggoner. 2015. "Are Samples Drawn from Mechanical Turk Valid for Research on Political Ideology." Research \& Politics 2(4):doi:10.1177/2053168015622072.

Cornesse, Carina and Annelies G. Blom. 2020. "Response Quality in Nonprobability and Probability-Based Online Panels." Sociological Methods \& Research online first:https://doi.org/10.1177/0049124120914940. doi: https://doi.org/10.1177/0049124120914940.

Eagle, Jeremy. 2020. "How Has the Coronavirus Changed How You Use the Internet?" Pp. https://www.nytimes.com/2020/04/09/learning/how-has-the-coronaviruschanged-how-you-use-the-internet.html in New York Times. New York City: New York Times.

Evans, MDR and Jonathan Kelley. 2011. "Us Attitudes Towards Human Embryonic Stem Cell Research." Nature Biotechnology 29(6):484-88. doi: 10.1038/nbt.1891.

Hossain, Mohammed Mahbub. 2020. "Current Status of Global Research on Novel Coronavirus Disease (Covid-19): A Bibliometric Analysis and Knowledge Mapping." SSRN: https://ssrn.com/abstract $=3547824$ 2020(May 18):http://dx.doi.org/10.2139/ssrn.3547824.

Hou, Tianya, Taiquan Zhang, Wenpeng Cai, Xiangrui Song, Aibin Chen, Guanghui Deng and Chunyan Ni. 2020. "Social Support and Mental Health among Health Care Workers During Coronavirus Disease 2019 Outbreak: A Moderated Mediation Model." PLoS One May 29, 2020:https://journals.plos.org/plosone/article?id=10.1371/journal.pone.0233831. doi: 10.1371/journal.pone.0233831.

Imhoff, Roland and Pia Lamberty. 2020. "A Bioweapon or a Hoax? The Link between Distinct Conspiracy Beliefs About the Coronavirus Disease (Covid-19) Outbreak and Pandemic Behavior." Social Psychological and Personality Science in press:forthcoming.

Lehmiller, Justin J., Justin R. Garcia, Amanda N. Gesselman and Kristen P. Mark. 2020. "Less Sex, but More Sexual Diversity: Changes in Sexual Behavior During the Covid-19 Coronavirus Pandemic." Leisure Sciences June 26, 2020:https://doi.org/10.1080/01490400.2020.1774016. doi: DOI: $10.1080 / 01490400.2020 .1774016$.

Likert, Rensis. 1932. "A Technique for the Measurement of Attitudes." Archives of Psychology, Health \& Medicine 22(1):5-55. doi: (not available) 
Manzo, Gianluca. 2020. "Complex Social Networks Are Missing in the Dominant Covid-19 Epidemic Models." Sociologica 14(1):31-49, $<$ https://sociologica.unibo.it/article/view/10839>. doi: https://doi.org/10.6092/issn.1971-8853/10839.

Matthewman, Steve and Kate Huppatz. 2020. "A Sociology of Covid-19." Journal of Sociology online before print:10.1177/1440783320939416. doi: 10.1177/1440783320939416.

McFadden, SarahAnn M., Amyn A. Malik, Obianuju G. Aguolu, Kathryn S. Willebrand and Saad B. Omer. 2020. "Perceptions of the Adult Us Population Regarding the Novel Coronavirus Outbreak." PLoS One 15(4):e0231808. doi:

https://doi.org/10.1371/journal.pone.0231808.

Melbin, Murray. 1978. "Night as Frontier." American Sociological Review 43(1):3-22.

Nowak, Piotr B. 2019. "Moment-Type Estimation from Grouped Samples." Statistics \& Probability Letters 149(June):80-85. doi: 10.1016/j.spl.2019.01.010.

Pennycook, Gordon, Jonathon McPhetres, Bence Bago and David G. Rand. 2020, April 14. "Predictors of Attitudes and Misperceptions About Covid-19 in Canada, the Uk, and the USA." edited by U. o. R. C. Hill/Levene Schools of Business. Hill/Levene Schools of Business, University of Regina (Canada): files.osf.io.

Schaeffer, Katherine. 2020. "Nearly Three-in-Ten Americans Believe Covid-19 Was Made in a Lab. ." PEW Fact Tank. 2020, April

8:https://www.pewresearch.org/facttank/2020/04/08/nearly-three-in-tenamericans-believe-covid-19-was-made-in-a-lab/.

Springer, Victoria A., I. Stephanie Vezich, Samuel C. Lindsey and Peter J. Martini. 2016. "Practice-Based Considerations for Using Multi-Stage Survey Design to Reach Special Populations on Amazon's Mechanical Turk." Survey Practice 9(5):1-10.

Treiman, Donald J. 2009. "Scale Construction." Pp. 241-62 in Quantitative Data Analysis. San Francisco: Jossey-Bass.

Varshney, Mohit, Jithin T. Parel, Neeraj Raizada and Shiv K. Sarin. 2020. "Initial Psychological Impact of Covid-19 and Its Correlates in Indian Community: An Online (Feel-Covid) Survey. ." PLoS One 15(5)(5):e0233874. doi: https://doi.org/10.1371/journal.pone.0233874.

Wang, Huiyao, Qian Xia, Zhenzhen Xiong, Zhixiong Li, Weiyi Xiang, Yiwen Yuan, Yaya Liu and Zhe Li. 2020. "The Psychological Distress and Coping Styles in the Early Stages of the 2019 Coronavirus Disease (Covid-19) Epidemic in the General Mainland Chinese Population: A Web-Based Survey." PLoS One 15(5):e0233410. doi: https://doi.org/10.1371/journal.pone.0233410.

Weinberg, Jill D., Jeremy Freese and David McElhattan. 2014. "Comparing Data Characteristics and Results of an Online Factorial Survey between a Population-Based and a Crowdsource-Recruited Sample." Sociological Science 1(1):292-310.

Yan, Youpei, Amyn A Malik, Jude Bayham, Eli P Fenichel, Chandra Couzens and Saad B Omer. 2020. "Measuring Voluntary and Policy-Induced Social Distancing Behavior During the Covid-19 Pandemic." Pp. Posted August 01, 2020. in medRxiv: COVID-19 SARS-CoV-2 preprints 
Zerubavel, Eviatar. 1985. Hidden Rhythms: Schedules and Calendars in Social Life. Berkeley and Los Angeles: University of California Press. 